Int. J. Dev. Biol. 57: 753-757 (2013)

doi: $10.1387 / \mathrm{ijdb} .130155 \mathrm{mb}$

\title{
Clonal analyses in the anterior pre-placodal region: implications for the early lineage bias of placodal progenitors
}

\author{
SUJATA BHATTACHARYYA and MARIANNE E. BRONNER* \\ Division of Biology, California Institute of Technology, Pasadena, CA, USA
}

\begin{abstract}
Cranial ectodermal placodes, a vertebrate innovation, contribute to the adenohypophysis and peripheral nervous system of the head, including the paired sense organs (eyes, nose, ears) and sensory ganglia of the Vth, VIlth, IXth and Xth cranial nerves. Fate-maps of groups of cells in amphibians, teleosts and amniotes have demonstrated that all placodes have a common origin in a horseshoe shaped territory, known as the preplacodal region (PPR), which surrounds the presumptive neural plate of the late gastrula/early neurula stage embryo. Given the extensive regional overlap of progenitors for different placodes in the chick embryo, it has been a matter of debate as to whether individual cells in the PPR are truly multipotent progenitors, with regard to placodal identity, or rather are lineage-biased or restricted to a specific placodal type prior to overt differentiation. Utilizing clonal analyses in vivo, we demonstrate here that the anterior PPR comprises some precursors that contribute either to the olfactory or lens placode well before they are spatially segregated or committed to either of these placodal fates. This suggests that lineage bias towards a specific placodal fate may coincide with induction of the PPR.
\end{abstract}

KEY WORDS: placode, olfactory, lens, pre-placodal region, lineage

Cranial placodes are transient ectodermal thickenings that are induced in localized regions of the embryonic head. The derivatives of the cranial sensory placodes include the lens of the eye, the olfactory epithelium of the nose and the inner ear. Given the seminal contributions that placodes make to the peripheral nervous system, their developmental origin has been examined in much detail. In particular, dye labeling small populations of cells in chick and Xenopus embryos has revealed that all placodes originate within a horseshoe-shaped domain that surrounds the anterior neural plate in early neurula stage embryos, termed the pre-placodal region (PPR) (Bhattacharyya et al., 2004; Pieper et al., 2011; Streit, 2002; Xu et al., 2008; reviewed in McCabe and Bronner-Fraser, 2009). However, within this domain, anteriorposterior regionalization relegates adenohypohyseal, olfactory and lens precursors to the anterior third of the PPR (known as the extended anterior placodal area: Toro and Varga, 2007) while the middle third is occupied mainly by trigeminal with a few otic precursors and finally the posterior third contains mostly precursors to the otic placode and all precursors of the epibranchial placodes. Despite this regionalization, focal dye injections often label adjacent placodes. While placode cells are multipotent, capable of giving rise to various cell types including neurons, hair cells, secretory cells and supporting cells amongst others, it has been unclear whether individual cells in the PPR can contribute to more than one type of placode. To address this question, we dye injected single cells in the anterior PPR and found that individual clones contribute to either the olfactory or lens placode (at early stages they also were able to contribute to either the epidermis or neural epithelium) but we were unable to detect any clones that spanned both the olfactory and lens placodes. While we cannot rule out the possibility that there are indeed precursors in the chick PPR that contribute to more than one type of placode, our results raise the intriguing possibility that lineage bias towards a particular placodal fate occurs concomitantly with demarcation of the PPR.

\section{Results}

Our population fate-map analyses in the early chick embryo established that olfactory and lens placode precursors show extensive overlap in the anterior PPR at neurula stages (Hamburger-Hamilton stage $6(\mathrm{HH} 6)$ ), with domains that contain either olfactory or lens precursors beginning to segregate away from each other at slightly

Abbreviations used in this paper: PPR, preplacodal region.

\footnotetext{
*Address correspondence to: Marianne E. Bronner. Division of Biology, 139-74, California Institute of Technology, Pasadena, CA 91125, USA. e-mail: mbronner@ caltech.edu
} 
later stages (HH8) (Bhattacharyya et al., 2004). In order to determine the fate of single cells at each of these time-points, individual precursors within the anterior PPR were iontophoretically injected with rhodamine dextran at $\mathrm{HH} 6$ and $\mathrm{HH} 8$ (Fig. 1A). Two criteria were used to verify single cell labeling. First, voltage measurements were carried out during penetration of the cell membrane, revealing a characteristic drop in membrane potential. Second, visualization under a fluorescence microscope was performed to confirm that only a single cell was labeled. Embryos were then allowed to develop for 12 to 48 hours. Typically, this resulted in clone sizes ranging from 50-100 labeled cells. The results show that, at HH8 ( $n=65$ surviving embryos), clones derived from a single cell contribute exclusively to the olfactory epithelium (Fig. 2 C-C"), the lens (Fig. 2 F-F"), the forebrain or the ectoderm (data not shown). In sections through the olfactory epithelium, rhodamine dextran can be seen in olfactory sensory neurons, marked by their expression of neurofilament and $\beta$ III-tubulin (Fig.2 D,D',E). These results suggest that olfactory and lens precursors are already segregated in terms of prospective placodal fate by $\mathrm{HH} 8$.

This raises the intriguing prospect of a lineage decision having occurred at a prior stage. To address this possibility, we performed an analogous investigation in $\mathrm{HH} 6$ (neurula stage) embryos $(\mathrm{n}=31$ surviving embryos), the earliest stage accessible to in ovo lineage analysis. The results show that even single cells injected at $\mathrm{HH} 6$ are restricted in their ability to contribute to one or the other placode. Interestingly, however, more medial injections (performed closer to the midline of the embryo) give rise to cells in the forming olfactory placode (Fig. 2 A-A") and in the forebrain. Slightly more lateral injections produce cells in the lens and neighboring ectoderm (Fig. 2 B-B"). These results show that cells in the anterior ectoderm of $\mathrm{HH} 6$ embryos can contribute to more than one embryonic cell type. In spite of this bipotency, we did not find clones that contribute to multiple manners of placode. It should be noted that the region closest to the tip of the prechordal plate predominantly contains nasal precursors while the posterior-most fourth of the shared lens/ olfactory domain is populated almost exclusively by lens precursors (Bhattacharyya etal., 2004). Therefore, it is possible that some of our injections targeted these already partitioned domains of ectoderm. Furthermore, we saw a 2:1 ratio of clones that contributed to the olfactory as opposed to the lens placode across stages; however, this may simply reflect a tendency to inject closer to the midline rather than any biological difference in the numbers of olfactory versus lens progenitors. Nonetheless, our most parsimonious conclusion is that some individual placode progenitors within the
A

\section{Experimental design}

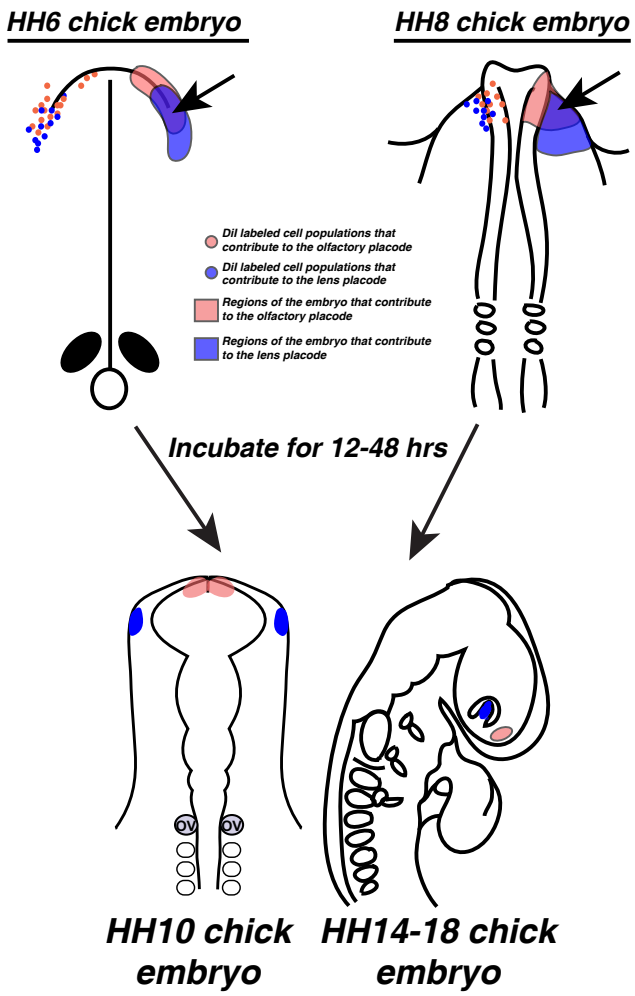

B

\section{Possible outcomes}
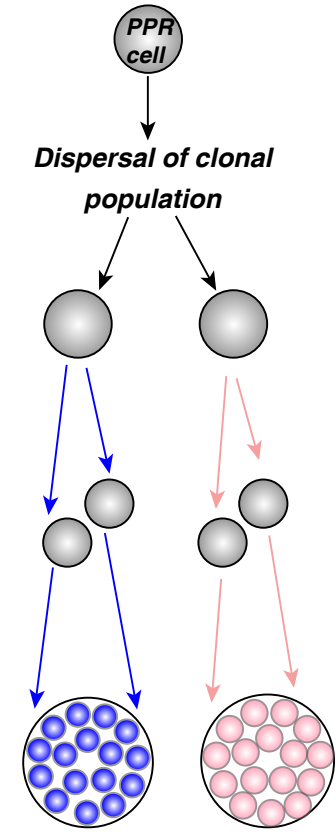

Lens placode

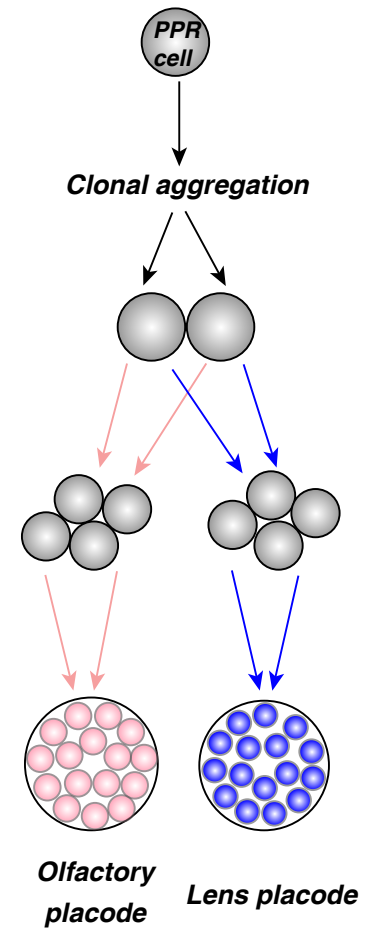

Fig. 1. Experimental design and hypothesis. (A) Experimental design. Black arrows point to the area targeted for injections in HH6 and HH8 chick embryos respectively. Pink dots/regions demarcate areas of the embryos where injecting small populations of cells results in labeling in the olfactory placode. Similarly, blue dots/regions correspond to labeled areas that contribute to the lens placode. Significant overlap is seen between these regions (3). Embryos were collected 12-48 hours later at which time olfactory and lens placode territories had completely separated (HH10) and were morphologically identifiable as placodes (HH14-18). (B) Possible outcomes. Individual progenitor cells in the PPR (PPR cell) can either divide and disperse to contribute to two different placodes, or can be lineage-biased and undergo minimal cell mixing to contribute to a single placode. Our data suggest that the latter is the case. 
anterior PPR are fated to contribute to a particular placodal type as early as the neurula stage. Interestingly, clones of cells derived from a single cell at either stage do not disperse throughout the area but rather form compact streams of cells even after the several rounds of cell division and fairly vigorous morphogenetic movements that occur (Fig. 1B).

\section{Discussion}

Given the extensive co-labeling of both the lens and olfactory placodes, when small groups of cells were labeled in the anterior chick PPR (Bhattacharyya et al., 2004), we expected this propensity for dual contribution to be mirrored at the level of individual cells. However, a recent study in Xenopus, mathematically deduced that the co-labeling of adjacent placodes by a single dye injection marking 5-15 cells is in fact "compatible with the possibility that adjacent placodes originate from non-overlapping regions of the PPR at neural plate stages" (Pieper et al., 2011). Our single cell lineage analyses in the chick embryo support the idea that focal injections could indeed label a heterogeneous population of differentially lineage biased placode precursors at the neurula stage albeit we lack sufficient statistical power in our data to rule out the possibility that there may be PPR cells whose progeny contribute to more that one placode. Interestingly, single cell lineage analyses in gastrula stage zebrafish embryos also reveals early fate restriction - progeny of anterior placodal progenitors mostly contribute either to the olfactory epithelium, lens or the pituitary (Dutta et al., 2005; Whitlock and Westerfield, 2000).

A key question that remains unresolved is whether individual cells within the PPR are in fact lineage-restricted and show targeted movements to their final destination or rather randomly change neighbors and acquire their fate based on

Fig. 2. Single cells in the pre-placodal region (PPR) are lineage-biased to an olfactory or lens placode fate. (A-A") Example of an embryo in which a single cell was labeled at $\mathrm{HH} 6$ and whose progeny contributes to the olfactory territory. (A) Bright field image of embryo collected at HH10. The rhodamine dextran labeled stream of cells is clearly visible in $A^{\prime}$ (arrow) and an overlay of the two images is presented in A." Dotted lines demarcate the presumptive forebrain of the embryo at this stage. (B-B") Example of an embryo collected at HH14 in which a single cell was labeled at HH6 and whose progeny contributes to the lens and adjacent ectoderm. (B) Bright field image; (B') Fluorescent image; (B") Overlap of the bright field and fluorescent images. Arrows in $B^{\prime}$ and $B^{\prime \prime}$ point to labeled cells in the lens. (C-C") Example of an embryo injected at HH8 and collected at HH18. A cluster of cells is clearly labeled within the olfactory epithelium. (C) Bright field image; $\left(\mathbf{C}^{\prime}\right)$ Fluorescent image; (C") Overlay. Dotted lines demarcate the eye and the olfactory placode in the head of the embryo. Arrows indicate labeled cells in the olfactory placode. (D-D") Example of an embryo collected at HH15/16 in which a single cell was labeled at HH8. Rhodamine dextran is clearly visible in the lens. (D) Bright field image. (D,E) Adjacent sections through embryo depicted in (C-C"). Blue: DAPl; Green: Neurofilament and beta-tubulin staining; Red: rhodamine dextran. (D') Same section as shown in (D) without $D A P$ I staining. Arrows in $D, D^{\prime}$ and $E$ point to a neuron in the olfactory epithelium born from the rhodamine labeled single cell. Dotted lines denote the basement membrane of the olfactory epithelium. (F-F") Example of an embryo injected at HH8 and collected at HH16 in which the clone contributes to the lens of the eye (arrows). (F) Bright field image; (F') Fluorescent image; (F") Overlay. ov: optic vesicle; e: eye; op: olfactory placode. local gradients of signaling cues. One striking observation in this study is that all clones derived from cells injected at either stage are found in a reasonably tight cluster as opposed to dispersing throughout the ectoderm. This is also compatible with the Xenopus study wherein no evidence was found for large-scale cell sorting when tracking the movement of single cells in a field of labeled placodal precursors; however, cells appeared to move as a coherent group within the ectoderm with local rearrangements contributing to limited cell mixing (Pieper et al., 2011). While the present study has not exhaustively labeled every cell in the anterior PPR, when taken together with our population fate-map, it suggests that there is likely to be an intermingling of precursors, which would argue for a need for small-scale cell sorting perhaps guided by signals in the microenvironment. Notably, local differences in the availability of Fgf4 and in the responsiveness of individual cells within the inner cell mass of the early mouse blastocyst results in a "salt and pepper distribution" of lineage-biased precursors for the pluripotent epiblast and primitive endoderm (Kang et al., 2012).

At first glance, our data suggesting the presence of lineagebiased placodal progenitors appear to contradict several lines of evidence for a shared "ground state " of the PPR. These include 1) a unique transcriptional profile - placodal cells co-express and their derivatives show a functional requirement for the transcription factors Eya1, Six1 and Six4 (Schlosser, 2010; 2006; Streit, 2007), 2 ) only cells that have acquired generic placodal properties can
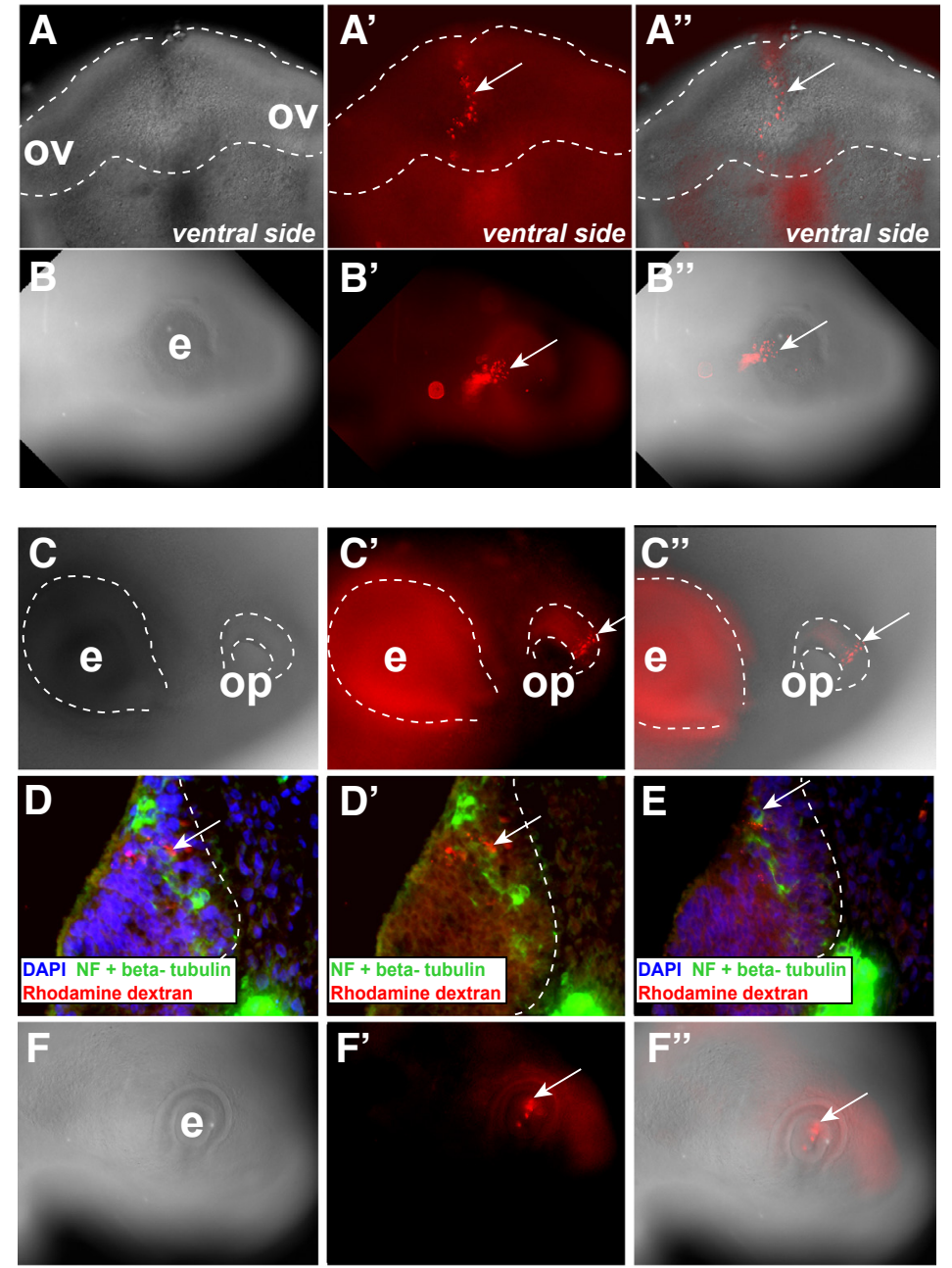
be induced to differentiate into specific placodal cell types (Martin and Groves, 2006) and 3) placodal precursors at all axial levels are specified as lens in an in vitro culture system (Bailey et al., 2006). However, increasing evidence points to cell-to-cell variation in gene expression, epigenetic and signaling responses in groups of cells previously thought to be homogenous. For example, both hematopoietic stem cell and embryonic stem cell populations are often heterogeneous in that individual cells express pluripotency genes unevenly and also show differential lineage-specific gene expression (reviewed in Enver et al., 2009). One possibility is that pluripotency in these systems is a meta-stable condition that might allow easier transition to a new cell state resulting in a more robust system supportive of highly regulative development (Silva and Smith, 2008). Consistent with this idea, developmental bias to contribute to either the inner cell mass or the trophectoderm has been observed as early as the 4-cell mouse embryo (Tabansky et al., 2013). While oriented cell divisions might be causal to this bias (reviewed in Zernicka-Goetz, 2005), it is currently thought that epigenetic factors (Torres-Padilla et al., 2007) and differing kinetics of Oct4 DNA binding (Plachta et al., 2011) may in fact perpetuate this bias.

Thinking of the PPR in a similar context, it is possible that stochastic fluctuations in gene expression perhaps in response to different signaling cues could result in an early compartmentalization of the ectoderm. We have shown previously that DIx5 and Pax6 are expressed at varying levels in the anterior ectoderm with some cells expressing higher levels of DIx5 and lower levels of Pax6 and vice versa (Bhattacharyya et al., 2004). Since DIx5 expression is ultimately strengthened in the olfactory placode but diminishes in the lens, it is possible that cells expressing initially higher levels of Dlx5 may be predisposed to an olfactory fate. In line with this hypothesis, DIx5 over-expressing cells are excluded from the lens (Bhattacharyya et al., 2004). Furthermore, DIx5 overexpression appears to alter the adhesive properties of cells such that all DIx5 expressing cells cluster together (Bhattacharyya et al., 2004). Thus, there could be internal and spatial constraints on multipotency resulting in lineage bias early in development. When these constraints are removed, either by placing the progenitors in an in vitro culture system (Bailey et al., 2006; Bhattacharyya and Bronner-Fraser, 2008) or by transplantation to a different environment in the embryo, the lack of commitment to a particular lineage is exposed (Bhattacharyya and Bronner-Fraser, 2008). Taken together, this suggests that the PPR may be a region of progenitors uniquely biased towards specific placodal fates despite sharing certain general properties. This premise deserves to be further explored by more extensive analyses of the fate of individual cells within the PPR coupled with live imaging to follow the cell movements of the clonal descendants. Technical advances in imaging and improved molecular and computational tools that allow single cells, their progeny and their interactions with their neighbors to be followed over time and space will facilitate such analyses (Dempsey et al., 2012; Sellmyer et al., 2013; Tabansky et al., 2013). Highlighting the significance of such an approach, a recent study utilizing in toto imaging of the zebrafish ventral neural tube, discovered that differentially specified progenitors intermingle with each other as a consequence of heterogeneous responses to a signaling cue prior to being sorted into distinctly defined progenitor domains (Xiong et al., 2013). The PPR might thus provide an excellent developmental context within which to interrogate the cellular and molecular mechanisms that result in the underlying variability that precedes the achievement of a stereotypic pattern of tissue growth and differentiation.

\section{Materials and Methods}

\section{Single cell injections}

Injecting electrodes were made from heat pulled aluminosilicate glass microcapillaries (A-M Systems) using a horizontal Sutter electrode puller. Electrodes were back-filled with $1 \mu \mathrm{l}$ of high molecular weight $(10 \mathrm{kDa})$ rhodamine dextran. This does not pass through gap junctions and leak into neighboring cells; hence, it is required for unambiguously labeling a single cell. Just prior to injecting, the rhodamine dextran in the electrode was overlaid with $1.2 \mathrm{M} \mathrm{LiCl}$. The electrode is then placed in the electrode holder reservoir and the reservoir is similarly filled with $1.2 \mathrm{M} \mathrm{LiCl}$. Once the electrode is prepared, the egg is windowed and India ink diluted 1:20 is injected into the sub-blastodermal cavity to visualize the embryo. A ground electrode is placed in the egg white via a small hole in the shell. At this point, the injecting electrode is lowered into Ringer's solution bathing the embryo and an intact electrical circuit is evidenced by the trace observed on the oscilloscope. The resistance of the electrode is measured. A tip resistance on the order of 20-30 $\mathrm{M} \Omega$ is indicative of a sharp electrode and can be used to penetrate the cell membrane of a single cell. An increase in noise is noted as the electrode approaches the embryo. The electrode is "rung" by very quickly turning the capacitance knob. If the electrode enters a cell, a drop in voltage, ranging from $10-40 \mathrm{mV}$ is noted. The dye is then released into the cell by injecting $4 \mathrm{nA}$ of current for 5 seconds. The electrode is then quickly removed from the cell and the oscilloscope trace should return to its ground value. Signals were amplified using an intracellular amplifier (Axon Instruments) and electrical activity was observed on an oscilloscope (Hitachi). The success of the injection was verified using epifluorescence. Embryos were harvested 12 - 48 hours later and fixed in $4 \%$ paraformaldehyde overnight at $4^{\circ} \mathrm{C}$. Approximately $90 \%$ of the embryos survived 24 hours later (96 out of 131 embryos injected).

\section{Histology and immunofluorescence}

Fixed embryos were washed several times in PBS and then scored for labeled cells in the head region using an upright Zeiss Axiophot fluorescence microscope. Labeled embryos were photographed at 10X magnification using an Axiocam digital camera attached to the fluorescence microscope. Some of these embryos were then processed for cryosectioning. The embryos were equilibrated in PBS containing $5 \%$ and $15 \%$ sucrose before being embedded in $7.5 \%$ gelatin (300 bloom, Sigma) and 15\% sucrose. The embryos were sectioned at $10 \mu \mathrm{m}$ and the slides stored at $4^{\circ} \mathrm{C}$. For purposes of immunostaining, sections through embryos were degelatinised by soaking in PBS warmed to $42^{\circ} \mathrm{C}$ for $5-10$ minutes. Neurofilament $(1: 250)$ and $\beta$ III-tubulin were diluted in blocking buffer as the primary antibodies with a goat anti-mouse IgG Alexa 488 used as the secondary antibody to detect both primary antibodies. Several washes with PBS containing $0.1 \%$ Tween were carried out after each application of antibody. Slides were finally rinsed in distilled water before being mounted in Permount containing 10 $\mu \mathrm{g} /$ $\mathrm{ml}$ DAPI, a nuclear stain.

\section{Acknowledgments}

We are extremely grateful to Dr. Scott E. Fraser for lending his expertise in teaching SB the technique of intracellular injections. We would also like to thank Drs. Tatiana Hochgreb and Marcos Simões-Costa for their insightful comments and helpful discussions. This work was supported by R01 DE16459 to MEB.

\section{References}

BAILEY A P, BHATTACHARYYA S, BRONNER-FRASER M, STREIT A (2006). Lens specification is the ground state of all sensory placodes, from which FGF promotes 
olfactory identity. Dev Cell 11: 505-517.

BHATTACHARYYA S, BAILEY A P, BRONNER-FRASER M, STREIT A (2004). Segregation of lens and olfactory precursors from a common territory: cell sorting and reciprocity of DIx5 and Pax6 expression. Dev Biol 271: 403-414.

BHATTACHARYYA S, BRONNER-FRASER M (2008). Competence, specification and commitment to an olfactory placode fate. Development 135: 4165-4177.

DEMPSEY W P, FRASER S E, PANTAZIS P (2012). PhOTO Zebrafish: A Transgenic Resource for In vivo Lineage Tracing during Development and Regeneration. PLOS ONE 7: e32888.

DUTTA S, DIETRICH J-E, ASPÖCK G, BURDINE R D, SCHIER A, WESTERFIELD M, VARGA Z M (2005). pitx3 defines an equivalence domain for lens and anterior pituitary placode. Development 132: 1579-1590.

ENVER T, PERAM, PETERSON C, ANDREWS P W (2009). Stem Cell States, Fates, and the Rules of Attraction. Cell Stem Cell 4: 387-397.

KANG M, PILISZEK A, ARTUS J, HADJANTONAKIS A-K (2012). FGF4 is required for lineage restriction and salt-and-pepper distribution of primitive endoderm factors but not their initial expression in the mouse. Development 140: 267-279.

MARTIN K, GROVES, A K (2006). Competence of cranial ectoderm to respond to Fgf signaling suggests a two-step model of otic placode induction. Development 133: 877-887.

MCCABE K L, BRONNER-FRASER M (2009). Molecular and tissue interactions governing induction of cranial ectodermal placodes. Dev Biol 332: 189-195.

PIEPER M, EAGLESON G W, WOSNIOK W, SCHLOSSER G (2011). Origin and segregation of cranial placodes in Xenopus laevis. Dev Biol 360: 257-275.

PLACHTA N, BOLLENBACH T, PEASE S, FRASER S E, PANTAZIS, P (2011). Oct4 kinetics predict cell lineage patterning in the early mammalian embryo. Nat Cell Biol 13: 117-123.

SCHLOSSER G (2006). Induction and specification of cranial placodes. Dev Biol 294: 303-351.

SCHLOSSER G (2010). Making senses development of vertebrate cranial placodes.
Int Rev Cell Mol Biol 283: 129-234.

SELLMYER M A, BRONSART L, IMOTO H, CONTAG C H, WANDLESS T J, PRESCHER J A (2013). Visualizing cellular interactions with a generalized proximity reporter. Proc. Natl. Acad. Sci. USA 110: 8567-8572.

SILVA J, SMITH A (2008). Capturing Pluripotency. Cell 132: 532-536.

STREITA (2002). Extensive cell movements accompany formation of the otic placode. Dev Biol 249: 237-254.

STREIT A (2007). The preplacodal region: an ectodermal domain with multipotential progenitors that contribute to sense organs and cranial sensory ganglia. Int $J$ Dev Biol 51: 447-461.

TABANSKY I, LENARCIC A, DRAFT R W, LOULIER K, KESKIN D B, ROSAINS J, RIVERA-FELICIANO J, LICHTMAN J W, LIVET J, STERN J N H, SANES J R, EGGAN K (2013). Developmental bias in cleavage-stage mouse blastomeres. Curr Biol 23: 21-31.

TORO S, VARGA Z M (2007). Equivalent progenitor cells in the zebrafish anterior preplacodal field give rise to adenohypophysis, lens, and olfactory placodes. Semin Cell Dev Biol 18: 534-542.

TORRES-PADILLA M E, PARFITT D E, KOUZARIDES T, ZERNICKA-GOETZ M (2007). Histone arginine methylation regulates pluripotency in the early mouse embryo. Nature 445: 214-218.

WHITLOCK K E, WESTERFIELD M (2000). The olfactory placodes of the zebrafish form by convergence of cellular fields at the edge of the neural plate. Development 127: 3645-3653.

XIONG F, TENTNER A R, HUANG P, GELAS A, MOSALIGANTI K R, SOUHAIT L, RANNOU N, SWINBURNE I A, OBHOLZER N D, COWGILL P D, SCHIER A F, MEGASON S G (2013). Specified neural progenitors sort to form sharp domains after noisy Shh signaling. Cell 153: 550-561.

XUH, DUDE CM, BAKER CVH(2008). Fine-grained fate maps for the ophthalmic and maxillomandibular trigeminal placodes in the chick embryo. DevBio/317: 174-186.

ZERNICKA-GOETZ M (2005). Cleavage pattern and emerging asymmetry of the mouse embryo. Nat Rev Mol Cell Biol 6: 919-928. 


\section{Further Related Reading, published previously in the Int. J. Dev. Biol.}

Patterning and cell fate in ear development

Berta Alsina, Fernando Giraldez and Cristina Pujades

Int. J. Dev. Biol. (2009) 53: 1503-1513

http://dx.doi.org/10.1387/ijdb.072422ba

The preplacodal region: an ectodermal domain with multipotential progenitors that contribute to sense organs and cranial sensory ganglia Andrea Streit

Int. J. Dev. Biol. (2007) 51: 447-461

http://dx.doi.org/10.1387/ijdb.072327as

The first steps towards hearing: mechanisms of otic placode induction

Takahiro Ohyama, Andrew K. Groves and Kareen Martin

Int. J. Dev. Biol. (2007) 51: 463-472

http://dx.doi.org/10.1387/ijdb.072320to

The neural induction process; its morphogenetic aspects

P D Nieuwkoop

Int. J. Dev. Biol. (1999) 43: 615-623

http://dx.doi.org/10.1387/ijdb.10668971

Developmental neurobiology of the anterior areas in amphibians: urodele perspectives G W Eagleson

Int. J. Dev. Biol. (1996) 40: 735-743

http://dx.doi.org/10.1387/ijdb.8877447

5 yr ISI Impact Factor $(2011)=2.959$
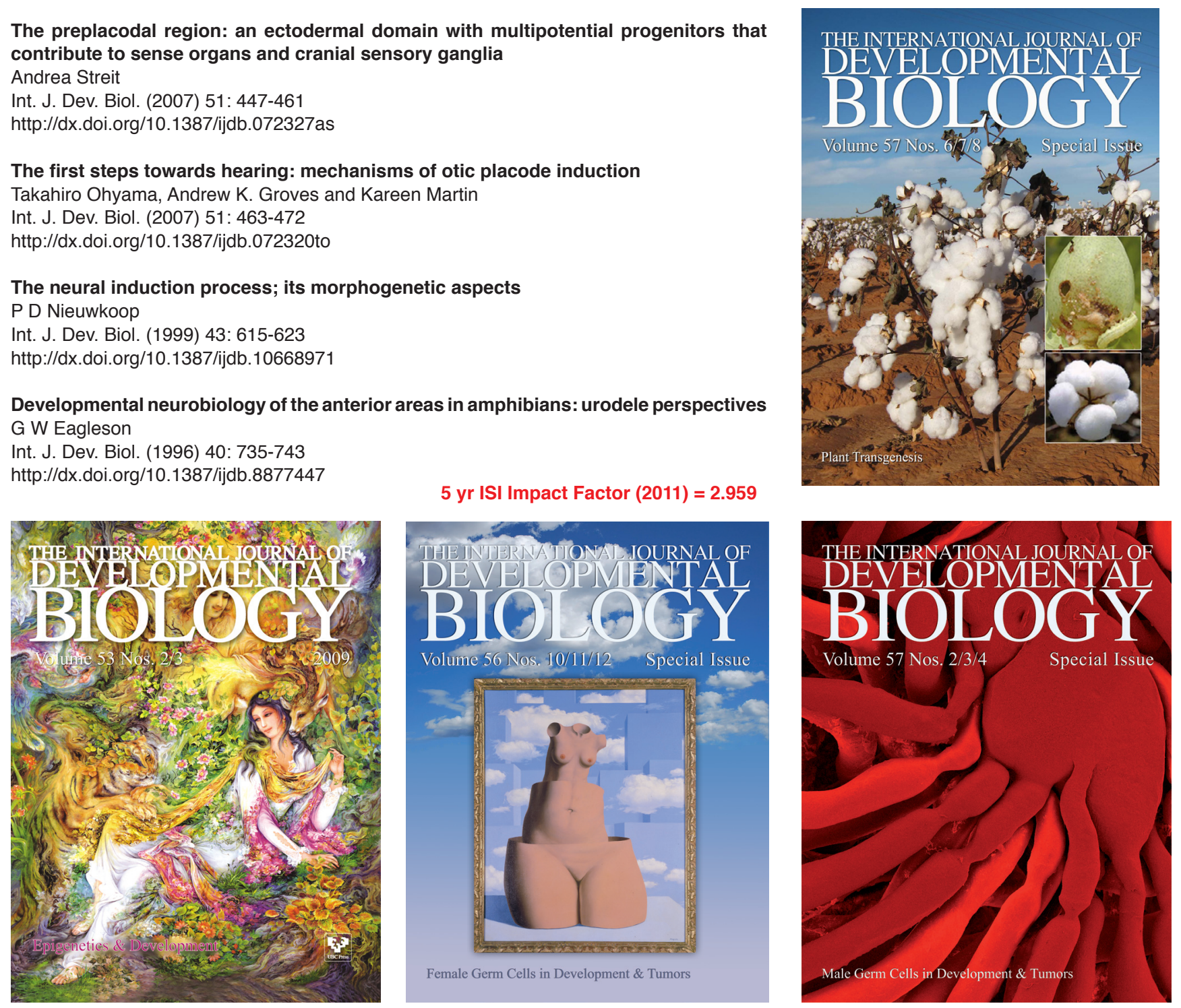